lower, current JP8 exposure. Significant associations were not observed between repeated-workday exposure to JP8 and neuropsychological performances.

Conclusions Results suggest that repeated JP-8 exposure, at levels not exceeding regulatory limits, does not significantly contribute to reduced neuropsychological proficiencies. We discuss potential explanations and implications for these findings.

\section{SENSITISATION TO RATS AND MICE AMONG LABORATORY STAFF AND RESEARCHERS}

Neda Dianati Maleki, Jeremy Beach, Harissis Vliagoftis, Eugene Waclawski, Nicola Cherry. University of Alberta, Edmonton, $A B$, Canada

\subsection{6/oemed-2014-102362.4}

Objectives To identify modifiable factors associated with sensitisation to laboratory animals.

Method Animal husbandry staff (group 1) and researchers (group 2) were recruited from a University animal facility together with health researchers not working with animals (group 3). Sensitisation was evaluated using skin prick tests to rat and mouse allergens. Current tasks, job history and demographic information were recorded.

Results The 3 groups comprised: 57; 57; and 50 subjects. Among group 1,88\% were currently working with rats, and $88 \%$ with mice: $51 \%$ were sensitised to rat, $28 \%$ to mouse. In group 2 the numbers exposed were lower ( $75 \%$ rat, $58 \%$ mice) as was the rate of sensitisation ( $32 \%$ rat, $12 \%$ mouse). No one in group 3 was exposed or sensitised. Among those currently exposed, sensitisation to rat was associated with shaving of fur and disposal of soiled litter, and with less frequent use of female rats. Sensitisation to mice was higher in those with contact with mouse urine and saliva, but not related to specific tasks. In multiple regression models, sensitisation to rat was only associated with use of female rats (OR $0.25,95 \%$ CI $0.01-0.64$ ). Sensitisation to mouse was greater in women (OR 6.67, 1.20-36.98), those born on a farm (OR 4.65, 1.05-20.55) and with exposure to mouse saliva (OR 4.26, 1.23-14.76).

Conclusions Exposures were highly correlated, making it difficult to identify specific modifiable risk factors. However it is of note that, since male rats use urine to mark territory, the greater use of exclusively female rats should serve to reduce sensitisation.

\section{MULTI-SITE MUSCULOSKELETAL PAIN AND PHYSICAL WORKING CONDITIONS AS PREDICTORS OF SICKNESS ABSENCE DUE TO MUSCULOSKELETAL DIAGNOSES}

\begin{abstract}
${ }^{1}$ Subas Neupane, ${ }^{1}$ Pekka Virtanen, ${ }^{2}$ Päivi Leino-Arjas, ${ }^{3}$ Helena Miranda, ${ }^{1}$ Anna Siukola, ${ }^{1}$ Clas-Håkan Nygård. 'School of Health Sciences, University of Tampere, Tampere, Finland; ${ }^{2}$ Finnish Institute of Occupational Health, Helsinki, Finland; ${ }^{3} \mathrm{OP}-$ Pohjola Group, Helsinki, Finland
\end{abstract}

\subsection{6/oemed-2014-102362.5}

Objectives To investigate the importance of multi-site musculoskeletal pain as a predictor of sickness absence days due to musculoskeletal disorders (MSD) among blue-collar employees, and to study what extent such a relationship might be confounded by physical loading at work.

Method Survey responses from 901 employees were linked to a food industry company's record of sickness absence due to MSD
( $\geq$ four days). Generalised Linear Models (GLM) with negative binomial distribution assumption was used in order to determine associations between the occurrence of multi-site pain (no pain, one-site and multi-site pain), individual variables, work related variables and sickness absence days due to MSD during a fouryear follow-up.

Results The high exposure group had about 92 and the low exposure about 72 all-cause sickness absence days yearly, and corresponding figures for absence due to MSD were 36 and 28. The share of MSD absence is about 40\% irrespective of the exposure. Single site pain did not predict absence, whereas multi-site pain turned out as an independent predictor. Multi-site pain predicts absence in the group with low biomechanical exposure, but not in the group with high exposure. The $p$-values for interaction show that the groups differ significantly both in case of repetitive movements and in case of awkward postures.

Conclusions This prospective cohort study revealed very high level of sickness absence in biomechanically strenuous work, represented by manual work in food industry. On average, the employees were absent over 80 calendar days, i.e. almost three months, yearly.

\section{HEALTH AND SAFETY IN COLLISION REPAIR SHOPS AFTER 2 YEARS OF PARTICIPATION IN COLLISION AUTO REPAIR SAFETY STUDY (CARSS)}

${ }^{1}$ Anca Bejan, 'David Parker, ${ }^{2}$ Lisa Brosseau, ${ }^{1}$ Maryellen Skan, ${ }^{1}$ Min Xi. ${ }^{1}$ Park Nicollet Institute, Minneapolis, MN, USA; ${ }^{2}$ University of Illinois, Chicago, IL, USA

\subsection{6/oemed-2014-102362.6}

Objectives This study evaluated the sustainability of health and safety improvements in small collision shops 1 year after completion of targeted intervention activities.

Method Workplace health and safety evaluations were conducted at baseline, after 1 year, and after 2 years. During Year 1, owners received quarterly phone calls, written reminders, safety newsletters, and had access to online services and in-person assistance with creating safety programs and respirator fit testing. During Year 2, owners received up to 3 postcard reminders regarding the services and resources available from study staff and website.

Results Forty-nine shops received baseline assessments, 45 were visited for 1-year follow-up and 33 were visited for 2-year follow-up. At baseline, the 33 shops had 19-60\% deficient items (mean $=45 \%$ items, SD $=10 \%$ ). At the end of Year 1 , there were $19-30 \%$ deficient items (mean $=27 \%$ items, $S D=11 \%$ ). At the end of Year 2, there were 11-37\% deficient items (mean $=27 \%$ items, $\mathrm{SD}=9.5 \%)$. Statistically significant changes $(\mathrm{p}<$ 0.05 ) from Year 1 to Year 2 were identified in three of the eight survey sections: compressed gasses ( $-8 \%$ deficient items), personal protective equipment $(-7 \%)$, and respiratory protection $(+6 \%)$. There was no difference in the magnitude of change in shops that received $0-2$ reminder cards vs. shops that received 3 cards.

Conclusions Results indicate that most business owners were able to maintain the health and safety improvements implemented during Year 1 of the CARSS intervention. The number of reminder cards sent to each business did not make a difference in the degree to which safety improvements were maintained. 